\title{
Une approche interdisciplinaire, pluri-échelle, multipartenaire des pollutions diffuses de l'eau : l'expérience de La Côte Saint-André (Isère)
}

\section{An interdisciplinary, pluriscale and pluripartner approach of water non- point-source pollutions : the La Côte Saint-André experience}

par F. Bel, A. Lacroix, A. Mollard,

Laboratoire d'Economie et de Sociologie Rurales, INRA/R\&A, Université Pierre Mendès France, Grenoble

C. David, N. Beaudoin, B. Mary

ISARA - Lyon, INRA - Laon, Unité d'Agronomie

G. Vachaud, M. Vauclin,

Laboratoire d'étude des Transferts en Hydrologie et Environnement,CNRS, INPG/Université Joseph Fourier, Grenoble

\section{B. Garino,}

Lycée d'Enseignement Général et Technique Agricole, La Côte-Saint-André

The Bievre-Liers plain $\left(300 \mathrm{~km}^{2}\right)$ surrounding the La Côte Saint André district represents a region of intensive agriculture where the nitrate concentration in the aquifer which regularly increases since the mid Seventies, threatens to go beyond the European limit of $50 \mathrm{mg} . \mathrm{l}$. Within this context a multidisciplinary and pluriannual scientific programme has been set up in 1991. By associating several academic research teams, local authorities as well as farmers, its objectives were oriented towards the development of an agriculture more concerned by groundwater quality, but still profitable. The paper presents a brief summary of the main results which have been obtained after a 4 yearperiod of intensive field work. Three spatial scales are considered :

- the field plot ranging from $1 \mathrm{~m}^{2}$ to $I$ ha for studying the detailed processes occuring in the soil-plant-atmosphere continuum, the soil being viewed as a bio-physico-chemical reactor,

- the farming cultivation system, to test different alternative less polluting scenarios,

- the entire plain for evaluating the impact of agricultural practice changes on the groundwater quality time evolution as well as to test the willingness of consumers to financially contribute to set up such alternative programmes.

\section{IINTRODUCTION}

\subsection{Rappel des objectifs}

Il s'agit d'étudier, à plusieurs échelles, les liaisons entre production agricole et protection de la ressource en eau souterraine en privilégiant les aspects analyse des processus et impacts économiques, dans une perspective d'agriculture durable.

Initiée en 1991 et s'inscrivant dans une action de longue durée, cette étude a déjà permis d'obtenir une bonne connaissance du milieu et d'identifier les problèmes posés par des techniques d'agriculture intensive sur la dégradation de l'environnement (manifestée essentiellement par la pollution en nitrates des eaux souterraines). Elle a également contribué à renforcer la connaissance de processus fondamentaux encore mal conceptualisés et à valoriser les acquis à plus grande échelle (le bassin versant), tant au niveau agro-économique qu'hydrogéologique, dans la perspective d'une agriculture durable et de la maîtrise de la ressource en eau souterraine.

De ce fait, l'approche mise en œuvre a regroupé deux volets :

i) un volet hydrologique mené à deux échelles différentes :

- à l'échelle stationnelle et parcellaire, une étude expérimentale et théorique concernant la compréhension et la modélisation (dans un but prédictif) des mécanismes prépondérants des transferts d'eau, de fertilisants et d'herbicides dans le système atmosphère-plante-sol-nappe et l'établissement de bilans précis, 
- à l'échelle régionale, une étude de l'évolution prévisible des ressources en eau souterraine (qualité et quantité) en fonction de l'utilisation des sols, de l'évolution et du climat. ii) un volet agro-économique développé à trois niveaux :

- l'expérimentation de scénarios alternatifs moins polluants à l'échelle de la parcelle et de l'exploitation,

- la simulation de programmes de préservation ou d'amélioration de la qualité de la ressource pour l'ensemble du bassin d'alimentation,

- enfin sur l'évolution prévisible de la demande en eau potable à long terme.

\subsection{Le site d'expérimentation et d'observation}

Le choix du site d'observation de la plaine de BièvreLiers s'explique à l'origine par la montée préoccupante, depuis une quinzaine d'années, des teneurs en nitrates dans les captages d'eau potable, certains d'entre eux dépassant la norme européenne de $50 \mathrm{mg}$ de $\mathrm{NO}_{3} / \mathrm{l}$; mais son intérêt réside aussi dans l'importance des synergies développées entre la recherche et les partenaires socio-économiques depuis 1991, date de mise en place d'un Observatoire sur les pollutions diffuses d'origine agricole dont le siège est situé au Lycée d’Enseignement Général et Technologique Agricole de La Côte-Saint-André.

\subsubsection{Intérêt hydro-géologique et économique}

Le bassin versant est caractérisé par un sol jeune filtrant (brun calcique ou brun calcaire) établi sur des alluvions d'âge wurmien à actuel et par la présence d'un système aquifère souterrain extrêmement puissant et complexe (deux nappes, fluvio-glaciaire et molassique), avec une très grande fragilité au niveau de la qualité de l'eau. Les sources de pollution responsables de la dégradation de la qualité de l'aquifère proviennent pour l'essentiel de pollutions diffuses, liées aux pratiques agricoles intensives, et secondairement de pollutions localisées induites par quelques cas de décharges en fond de carrière. En fait, bien qu'il existe en Rhône-Alpes des zones où les niveaux de pollution sont plus alarmants, la plaine de Bièvre-Liers est assez représentative de l'agriculture intensive de la région et constitue un très bon terrain pour la recherche de solutions non dictées par l'urgence, dans une perspective de développement durable.

\subsubsection{Intérêt au plan des synergies entre équipes et programmes de recherche}

Situé entre Grenoble et Lyon, le site atelier de La CôteSaint-André a permis la réalisation d'un grand nombre d'expérimentations mettant en situation de coopération interdisciplinaire des équipes appartenant à de nombreux organismes de recherche (CNRS, INRA, CEMAGREF, Universités, Ecoles d'Ingénieurs). Ceci a facilité de fortes interactions entre différentes communautés scientifiques : sciences de l'univers (hydrologie, hydrogéologie, physique du sol, géochimie, climatologie) ; sciences chimiques (chimie des xénobiotiques et des interfaces) ; sciences de la vie (microbiologie des sols, physiologie végétale, agronomie) et sciences de l'homme et de la société (économie). Grâce à l'accumulation des expérimentations et observations depuis 1991, le site est doté maintenant d'infrastructures importantes d'observation et constitue désormais une station de référence pour de nombreuses recherches, françaises et européennes. On trouvera la liste complète des laboratoires et chercheurs impliqués dans le programme dans le rapport final [1].

Ainsi, depuis l'origine, le programme en cours a pu bénéficier d'une reconnaissance :

- au niveau régional : Observatoire Rhône-Alpes des Pollutions Diffuses Agricoles, Ministère de I'Environnement DIREN, Ministère de I'Agriculture -DERF et Chambre Régionale d'Agriculture, Contrat de Plan Etat-Région,

- au niveau national : Programme Environnement, Société et Modes de Vie du CNRS (Comités SEAH et ESDLT), Zone Atelier du GIP HydrOsytèmes,

- au niveau européen : 3 contrats communautaires DGXII, programmes "STEP" et "Environnement 1992-1994".

\subsubsection{Intérêt au plan des interactions avec les partenaires socio-économiques}

Depuis 4 ans, on note une sensibilité croissante des agriculteurs aux résultats de la recherche, ce qui s'explique par le partenariat très actif développé par les équipes de recherches avec différents organismes professionnels et partenaires de développement :

- la mobilisation de la profession agricole sur le problème des pollutions diffuses a motivé l'inscription de la plaine de Bièvre-Liers dans les actions Fertimieux et la constitution d'un Comité de pilotage “ Pil'azote Bièvre " qui est un utilisateur potentiel des résultats de cette recherche. Cette initiative résulte du regroupement des partenaires socio-économiques suivants : la Chambre d'Agriculture de I'Isère, la Chambre Régionale d'Agriculture, l'Association Syndicale Autorisée d'Irrigation Bièvre-Liers, les Instituts techniques (ITCF et CETIOM) et les organismes professionnels tels que l'AGPM, avec le soutien de l'Agence de l'eau Rhône-Méditerranée-Corse et du Conseil général de I'Isère,

- ces divers organismes sont eux-mêmes impliqués aujourd'hui dans le projet “ Agr'eau”, projet pluriannuel de R \& D au niveau régional coordonné par la Section Environnement de la Chambre Régionale d’Agriculture. Ce projet est ciblé sur 4 zones-pilotes de la région et complété par des réseaux de parcelles expérimentales. Il est actuellement envisagé de mieux favoriser le tranfert de méthodologies et les échanges sur les résultats des recherches réalisées. Par ailleurs, la Chambre Régionale d'Agriculture est partenaire de la bourse de thèse qui a été attribuée pour trois ans dans le cadre de ce programme,

- le Lycée d'Enseignement Général et Technologique Agricole de La Côte-Saint-André est également partie prenante de ce projet avec deux types d'apports différents : l'appui technique de la ferme expérimentale pour la réalisation et le suivi des expérimentations et des mesures à l'échelle stationnelle et de la parcelle (étude des processus) ; la vulgarisation ou la formation à destination des agriculteurs (CFPPA), des étudiants et des enseignants. Le Lycée est enfin un lieu d'accueil des réunions d'information sur l'avancement de la recherche,

- enfin, dans la mesure où la recherche entreprise concerne au premier chef les usagers des services des eaux et les communes auxquelles ceux-ci sont rattachés, le contact a été noué avec deux organismes regroupant les collectivités territoriales concernées : le Syndicat d'aménagement de la plaine de Bièvre-Valloire (ex association Bièvre-ValloireAvenir) et le District de Saint Etienne de Saint Geoirs. Un accueil favorable a été apporté à la réalisation des enquêtes et des séances d'expérimentation destinées à évaluer le 
consentement à payer des consommateurs de la plaine de Bièvre pour la préservation de la qualité de l'eau potable.

\subsubsection{Présentation des résultats de recherche}

On présente dans deux parties successives une brève synthèse des résultats obtenus dans les volets hydrologique puis agro-économique. Un rapport final exhaustif présente de manière détaillée les résultats de recherche obtenus au terme de ces années de travail. Il inclut également deux types de publications : des thèses directement associées au programme (résumés) et des articles dont la plupart sont publiés dans des revues internationales de langue anglaise [1].

\section{II $\square$ HYDROLOGIE DU SOL : COMPRÉ- HENSION ET MODÉLISATION DES PROCESSUS}

Trois échelles spatiales d'investigation ont été considérées.

\subsection{L'échelle du lysimètre $\left(1,7 \mathrm{~m}^{3}\right)$}

L'échelle du lysimètre de sol non remanié implanté sur le site atelier (figure 1) permet l'étude fine des mécanismes d'écoulement, de transformation et de dégradation de l'azote et de l'atrazine, herbicide couramment utilisé sur maïs. La réalisation d'essais multi-traceurs en régime hydraulique permanent et le suivi spatio-temporel des molécules apportées (sous forme de créneaux de concentration) : traceurs isotopiques de l'eau, traceurs anioniques bio-transformables $\left(\mathrm{NO}_{3}{ }^{-}\right)$ou non $\left(\mathrm{Br}^{-}, \mathrm{Cl}^{-}, \mathrm{I}^{-}\right)$ cations échangeables $\left(\mathrm{NH}_{4}{ }^{+}\right)$ou non $\left(\mathrm{K}^{+}, \mathrm{Na}^{+}, \mathrm{Ca}^{++}, \mathrm{Mg}^{++}\right)$substance organique bio-dégradable (atrazine) ont mis en évidence les principaux résultats suivants $[2][3][4]$ :

\section{LEGENDE}

\begin{tabular}{|c|c|}
\hline & Sous - parcelle fertiliste (A) \\
\hline & Sous - parcele non fertliste (B) \\
\hline & Site fertlise + azote-15 \\
\hline & Essais doses (AGPM) \\
\hline$\square$ & Stations de mesure de bilans \\
\hline - & Points de pretivements gravimettriques \\
\hline $\mathbf{M}$ & Equipements micrometterologiques \\
\hline (L) & Lysimetres (Nord et Sud) \\
\hline $\mathbf{P}$ & Puits \\
\hline
\end{tabular}

1. Schéma des dispositifs expérimentaux mis en place sur le site atelier de La Côte-Saint-André.
- l'existence d'une fraction d'eau immobile (de l'ordre de $20 \%$ de la teneur volumique totale) invalidant les hypothèses sous-tendant l'approche classique de type convectiondispersion et nécessitant l'introduction d'une répartition bi-modale du champ de vitesse de pore avec une cinétique d'échange physique,

- une exclusion anionique (d'environ $10 \%$ en volume) due aux charges négatives de surface des argiles,

- la forte potentialité de nitrification du sol (tout l'ammonium apporté en $140 \mathrm{~h}$ a été transformé en nitrate pendant la durée de l'essai, soit 65 jours),

- un retard (par adsorption) et une perte importante (75\%) par biodégradation de l'atrazine. On notera néanmoins que l'existence d'écoulements préférentiels d'origine structurale peut conduire à un entraînement de l'atrazine
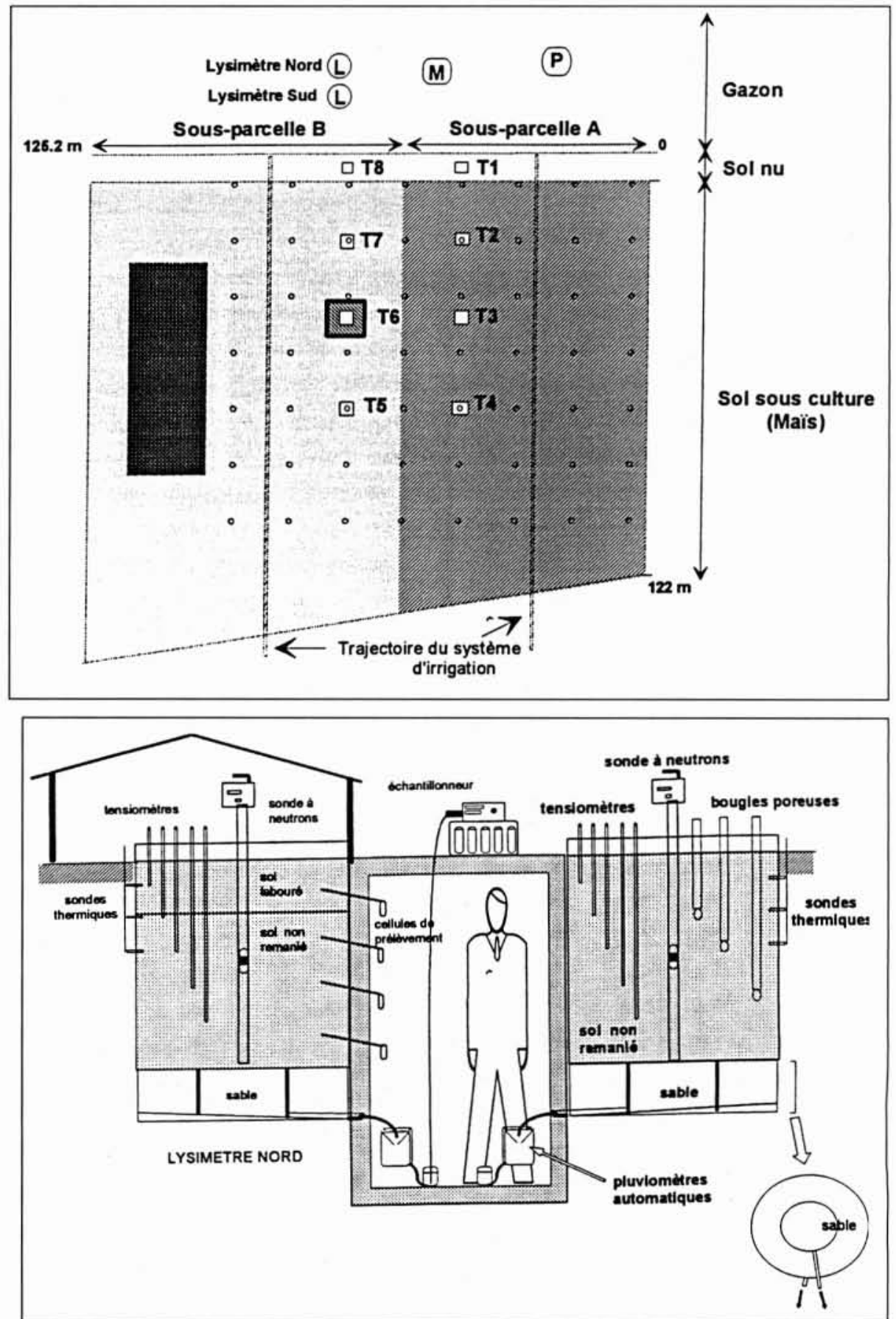
vers la profondeur (engendrant un risque de pollution des eaux souterraines), dès lors que le temps caractéristique du transport convectif dans la macroporosité est inférieur à ceux des phénomènes de fixation et de biodégradation, essentiellement prépondérants dans les couches superficielles du sol.

De plus, le dispositif expérimental a permis de valider l'estimation des flux hydriques par la loi de Darcy appliquée aux différentes stations de mesure du bilan hydrique et azoté implantées sur des parcelles d'essais (voir ci-dessous).

\subsection{L'échelle parcellaire (2x1 ha)}

- La métrologie non destructive mise en œuvre à cette échelle (humidimétrie neutronique pour le suivi hydrique dans la zone racinaire du maïs irrigué, tensiométrie pour la mesure de la pression capillaire, cellules poreuses de prélèvement de la solution du sol pour la mesure des concentrations en éléments chimiques) a permis un suivi fin pluriannuel des dynamiques et des bilans hydriques et azotés, sous différents traitements agronomiques, incluant le sol nu (figure 1) et pour des conditions climatiques très contrastées. Les principaux résultats sont exposés dans [5][6]. Les données sont disponibles sur le site Web http://lthe715.hmg. inpg.fr/lacote/ - L'utilisation d'apports d'engrais marqué en $15 \mathrm{~N}$ a permis d'effectuer des bilans azote détaillés. Il apparaît clairement [7] que les irrigations sont correctement valorisées par la culture et la lixiviation des nitrates négligeable durant le cycle cultural. En revanche, les pertes d'eau par percolation profonde qui se produisent à l'interculture (jusqu'à $90 \%$ des pluies) convectent les reliquats azotés présents dans le sol à la récolte. On a ainsi montré qu'une diminution de $80 \mathrm{~kg} / \mathrm{ha}$ d'apport en azote par rapport à la pratique courante de $260 \mathrm{~kg} / \mathrm{ha}$ réduisait drastiquement les pertes en nitrates au-delà de la zone racinaire sans pour autant affecter notablement la production (dont l'optimum est obtenu, d'après l'AGPM, pour $180 \mathrm{~kg} / \mathrm{ha}$ environ). On notera que ces résultats font l'objet d'un transfert vers le secteur aval puisque les agriculteurs de la région sont maintenant incités à adopter cette stratégie (suivis AGPM).

- Les données expérimentales acquises, complétées par des campagnes de caractérisation hydro-dispersive du sol [8] ont servi de support à la modélisation des transferts hydriques [9] et de nitrates [6]. A titre d'exemple, la figure 2 montre la comparaison entre les valeurs mesurées et calcu-
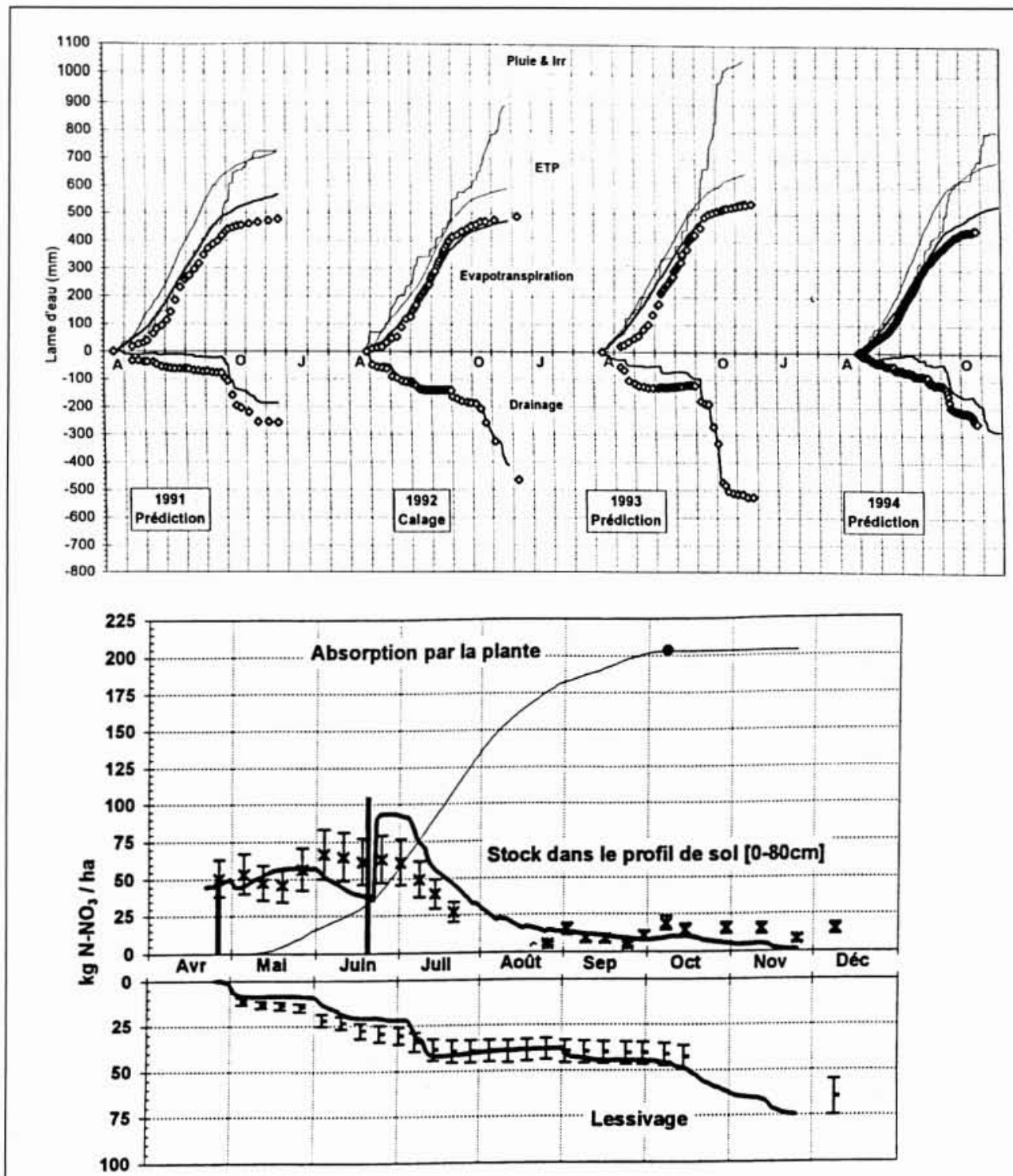

\section{Evaluation du} modèle WAVE.

En haut : principaux termes du bilan

hydrique, site cultivé fertilisé (T4).

Comparaison entre drainage et évaporation estimés et simulés.

En bas : Evolution temporelle du stock d'azote nitrique présent dans la zone racinaire, lessivage cumulé au-delà de cette zone, et absorption d'azote par la plante. Site cultivé fertilisé (T4), 1992. Comparaison entre mesures et simulations. 
lées par le modèle WAVE (Water and Agrochemicals in the soil, crop and Vadose Environment) des composantes du bilan hydrique, du stock de nitrate dans les 80 premiers centimètres du sol et des pertes par lixiviation.

- La non disponibilité de l'ensemble des paramètres nécessaires à la modélisation mécaniste des transferts et du transport conduit bien souvent à une phase de calage des modèles. Il a été montré tout l'intérêt de conduire des expérimentations parallèles sur sol nu et cultivé (avec et sans fertilisation) afin de discriminer les processus et de caler les paramètres sol et plantes indépendamment.

\subsection{L'échelle régionale du bassin versant de la Bièvre $\left(180 \mathrm{~km}^{2}\right)$}

Cette échelle est utile pour étudier l'effet des pratiques culturales actuelles sur la ressource en eau souterraine et prévoir son évolution future dans la perspective de modifica-

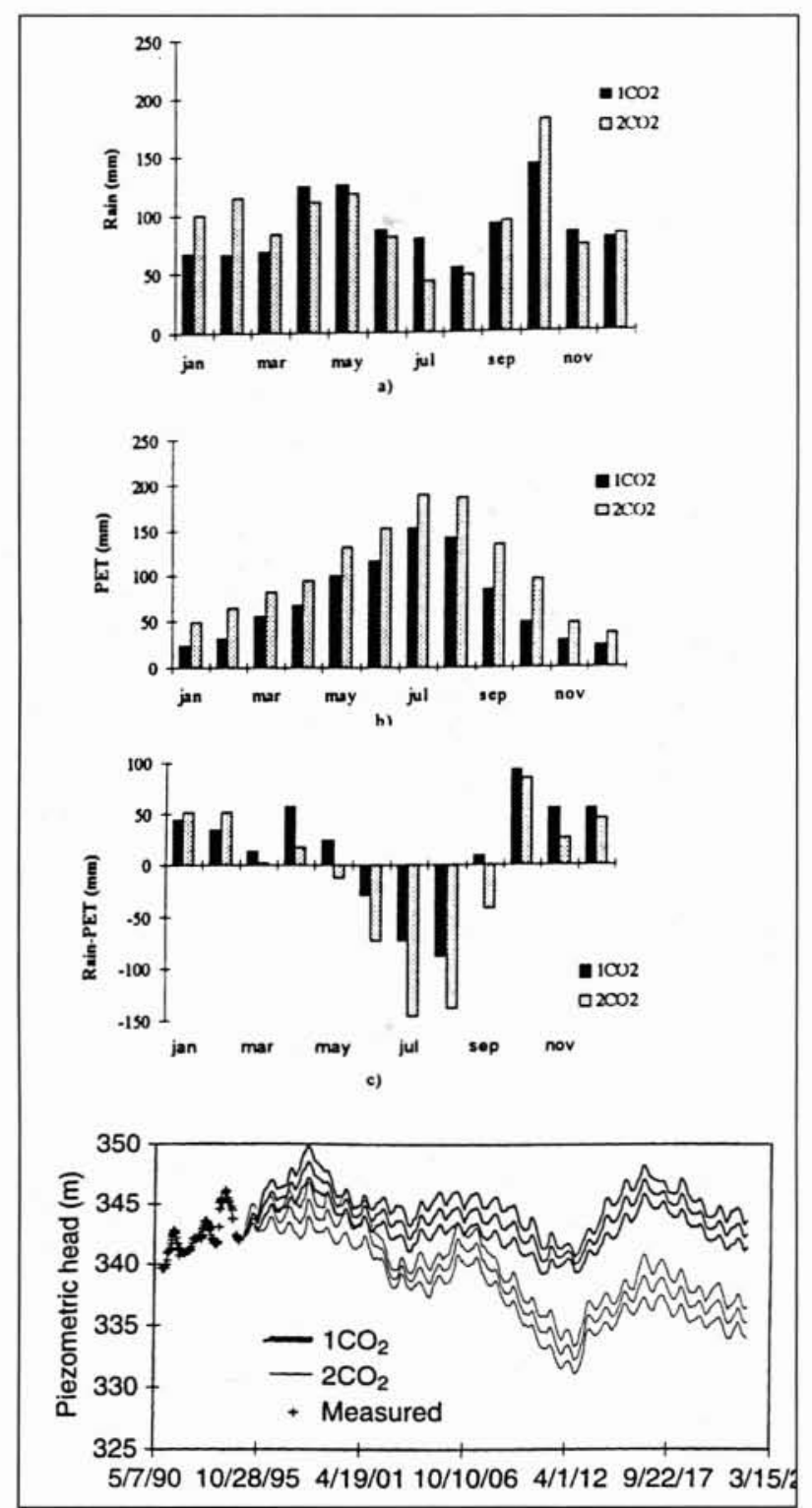

3. Impact d'un doublement du $\mathrm{CO}_{2}$ atmosphérique sur la pluviométrie, la demande évaporative de l'atmosphère et du déficit hydrique dans la région de La Côte-Saint-André et conséquence possible sur le niveau piézométrique de la nappe. tions anthropiques ou climatiques. A cette échelle, une modélisation temporellement continue et spatialement distribuée combinant un SIG (GRASS/mode raster en l'occurrence) et le modèle mécaniste ANSWERS (Areal Non point Source Watershed Environment Response Simulation [10]) - moins " gourmand " en paramètres que WAVE - a été développée pour simuler dans une première phase l'évolution spatio-temporelle des composantes du bilan hydrologique, en liaison avec les pratiques culturales. Elle s'appuie sur un Modèle Numérique de Terrain, sur la carte des sols et de la végétation, sur les champs spatiaux de précipitation et d'Evapotranspiration Potentielle et sur la piézométrie de l'aquifère. Cette approche a pu être validée :

- à l'échelle parcellaire par comparaison avec des valeurs d'évapotranspiration et drainage observées sur les parcelles en sol nu et sous culture de maïs irrigué, et ce, sur $\mathbf{3}$ années consécutives.

- à l'échelle du bassin versant sur la piézométrie de la nappe, mesurée en 5 puits différents.

Elle a été également utilisée à titre exploratoire pour prédire l'impact d'un changement climatique (scénario classique de doublement du $\mathrm{CO}_{2}$ atmosphérique) sur l'évolution de la ressource en eau souterraine. Pour ce faire, une méthode de désagrégation des sorties (pluies, température) du MCG du LMD a été proposée afin de les rendre spatialement compatibles avec le modèle hydrologique et de générer de longues séries temporelles (30 ans) climatiques [11].

Il apparaît qu'un tel scénario aurait un impact négatif sur la ressource en eau de cette région par la conjugaison d'une diminution de la recharge de l'aquifère (voir figure 3 ) et une augmentation des besoins en eau des cultures. Les résultats suggèrent également l'intérêt de considérer d'autres spéculations que le maïs, telles que le blé d'hiver, ou la luzerne, afin de mieux sauvegarder la ressource en eau.

\section{III — EXPÉRIMENTATION AGRONOMIQUE ET SIMULATION ÉCONOMIQUE DE PROGRAMMES PRÉVENTIFS PRÉ- SERVANT LA QUALITÉ DE L'EAU}

\subsection{Etat des lieux, méthodes d'observation et d'expérimentation}

En plaine de Bièvre-Liers, l'agriculture est l'activité économique dominante : elle s'étend sur près de 30000 ha de SAU, cultivés par un millier d'exploitations agricoles. Elle représente, aujourd'hui, le noyau dur de la production intensive et commerciale du département. Depuis les années 70 , l'intensification des systèmes de culture et d'élevage (extension du maiis au détriment des prairies, progression de la surface irriguée, accroissement des superficies en colza, tournesol, pois, tabac) explique en grande partie l'augmentation des teneurs en nitrates dans les captages d'eau potable.

On a mis en cuvre trois niveaux d'expérimentation et d'observation :

- des expérimentations à la parcelle, pendant deux campagnes successives (1996 et 1997) sur 4 exploitations agricoles pour évaluer et modéliser les pertes d'eau et d'azote sous différents systèmes de culture ; ces évaluations ont été complétées par d'autres références locales ou régionales.

- des enquêtes sur 20 exploitations (1996-1997) destinées à compléter les 4 monographies expérimentales pour 


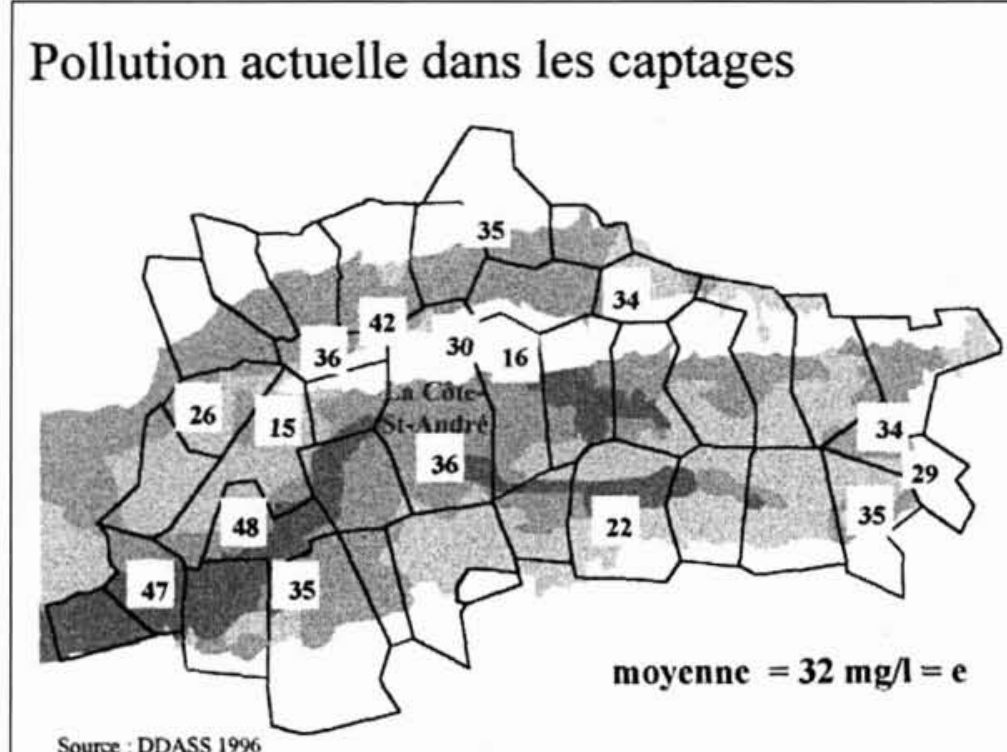

\section{Risque futur de pollution estimé sous les racines}

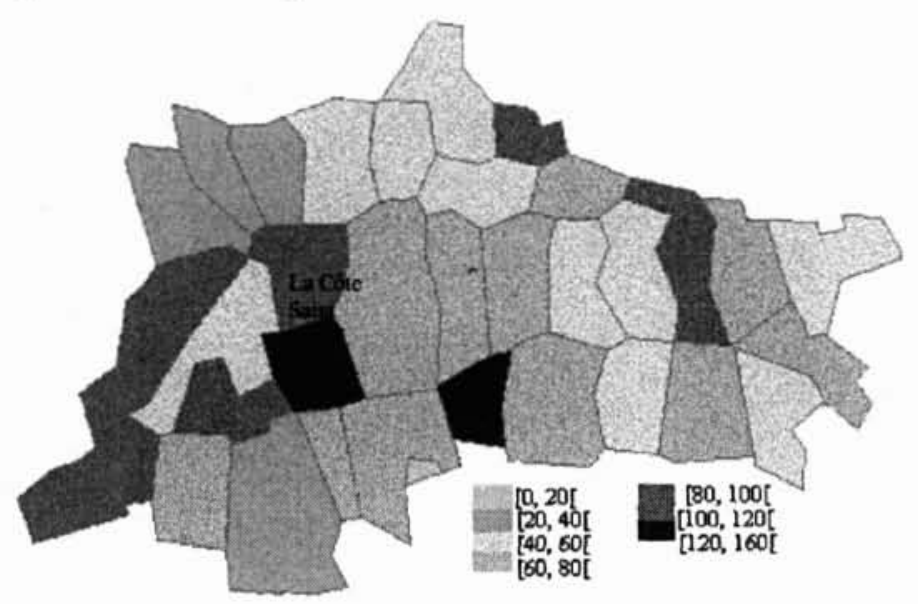

Source : INRA/R\&A 1995

moyenne $=64 \mathrm{mg} / \mathrm{l}=\mathrm{x}$

sions polluantes exprimé en kilos d'azote résiduels/ha sous les racines [13] et qui représente un potentiel de concentration moyenne en nitrates de la recharge de la nappe.

Selon cet indicateur, les fuites d'azote lessivable imputables à l'ensemble des exploitations de la plaine de BièvreLiers peuvent être estimées en moyenne à $54 \mathrm{~kg} / \mathrm{ha}$, soit 1610 tonnes d'azote par an. Les émissions polluantes émises par les industries, les communes et les forêts ont été évaluées à 303 tonnes d'azote par an, soit $16 \%$ de l'ensemble [1]. Au total, on peut considérer que ces fuites d'azote correspondent à une concentration moyenne de la recharge de la nappe de $\mathbf{6 4} \mathbf{~ m g}$ de $\mathrm{NO}_{3}$ par litre d'eau (1914 tonnes d'azote pour $132,7 \times 10^{6} \mathrm{~m}^{3}$ ). Cette évaluation dépasse nettement la norme de potabilité de $50 \mathrm{mg} / \mathrm{l}$ et représente le double des concentrations en nitrates constatées actuellement dans la partie inférieure de la nappe, où est prélevée l'eau potable (figure 4). Elle paraît cependant inférieure à des évaluations similaires réalisées par les agronomes sur d'autres sites [14] [15].

\subsection{Scénarios techniques et programmes de réduction des pollutions diffuses}

Dans un premier temps, les solutions curatives de traitement des eaux polluées (dénitrification, dénitratation) ont été évaluées [16]. Leur coût croît avec le niveau de pollution et est sensiblement plus élevé en zone rurale. Comparativement, les solutions préventives sont plus intéressantes car, à coût égal, elles sont cohérentes avec une agriculture durable [17]. Sur quelles solutions techniques fonder des programmes préventifs capables de réduire rapidement

mieux évaluer les pratiques culturales actuelles, les coûts et les difficultés de mise en œuvre de scénarios alternatifs et leur impact environnemental ;

- une base de données sur la quasi-totalité des exploitations de la plaine de Bièvre-Liers tirée des déclarations PAC (1994 et 1995) des exploitants et du fichier des quotas laitiers (807 exploitations). Cette base fournit les principales variables structurelles et permet d'évaluer les émissions polluantes de chaque exploitation pour les pratiques courantes de fertilisation.

Pour effectuer cette évaluation, nous adoptons l'approche des agronomes en termes de " systèmes de production polluants ", c'est-à-dire que le niveau d'émissions polluantes est fonction non seulement de la quantité d'engrais azotés consommée, mais beaucoup plus largement de la structure de la production, de l'importance de l'élevage, des successions culturales et des pratiques cumulées de fertilisation [12]. Sur cette base nous avons mis au point un indicateur d'émis- les fuites résiduelles à un niveau tel qu'à l'horizon de transfert, la pollution ambiante retrouve un niveau inférieur à la norme de potabilité ? La réponse des agronomes, pour l'essentiel, consiste à modifier la gestion de l'azote et de I'interculture. Parmi les différents scénarios techniques qui s'inscrivent dans cette voie (fertilisation raisonnée, cultures intermédiaires, successions culturales, diversification), seuls les plus simples à mettre en œuvre ont été expérimentés en 1995 et 1996 sur les 4 exploitations volontaires. Leur faisabilité technique et économique et leur impact sur la réduction des pollutions ont été également testés sur les 20 exploitations objets de l'enquête. On a ainsi défini trois pro-

1 En effet, selon les données DDASS concernant 22 captages, la concentration moyenne s'élevait à $32.5 \mathrm{mg} / \mathrm{l}$ en 1995, avec une grande variabilité selon les captages ( 16 à $48 \mathrm{mg} / \mathrm{l})$. 
grammes préventifs comme base possible d'une politique publique de la qualité de l'eau souterraine.

- le programme FARM (Fertilisation Ajustée sur le Rendement Moyen) consiste à limiter les objectifs de rendement à la moyenne des 5 dernières années et à optimiser la fertilisation azotée, y compris la gestion des effluents d'élevage, par rapport à cet objectif. Ce scénario de base minimal doit être appliqué à toute entreprise pour réduire les pratiques de surfertilisation. Son coût est modéré pour les entreprises agricoles.

- le programme FARM+CIPAN cumule le précédent avec la mise en place systématique de " cultures intermédiaires pièges à nitrates " avant les cultures de printemps et une meilleure gestion des résidus de récoltes (broyage/enfouissement des cannes de maïs). Il permet de réduire les fuites d'azote pendant l'interculture. Il induit des contraintes techniques et de travail qui se répercutent plus fortement sur les coûts des entreprises.

- le programme DISC est ciblé sur la " diversification des systèmes de cultures ". Il est plus complexe à mettre en œuvre que les deux précédents et plus difficile à expérimenter. En effet, il nécessite une modification importante des structures de production avec une réduction des cultures de printemps, notamment de la culture du maïs et, éventuellement, l'introduction de prairies en cas d'élevage. C'est un scénario certainement coûteux mais qui garantit une réduction significative et durable de la pollution azotée. Son impact technique et économique est encore insuffisamment évalué.

\subsection{Analyse coût-efficacité de programmes préventifs au niveau du bassin versant}

L'analyse coût-efficacité permet de rechercher quelle réduction des pollutions nitriques peut être attendue des programmes préventifs et à quels coûts. Grâce à la base de données, on a pu évaluer pour chaque exploitation l'indicateur d'émissions polluantes. Par simple agrégation, et sous l'hypothèse d'émissions non agricoles constantes, on a donc simulé pour l'ensemble du bassin versant l'impact global des 2 programmes les mieux référencés en terme de réduction des émissions polluantes ${ }^{2}$. Il s'agit bien d'une simulation, c'està-dire que les entreprises sont supposées capables d'adopter sans difficulté les solutions techniques envisagées et que leur efficacité est censée être maximale sans délai, ce qui ne serait évidemment pas le cas dans la réalité et nécessiterait la mise en œuvre d'un système d'incitations. Les recherches se poursuivent actuellement sur un tel système.

Quels coûts sont pris en compte ? Les coûts directs des entreprises pour mettre en œuvre les programmes préventifs. Pour le programme FARM, ce sont les pertes de recettes dues à une baisse des rendements, déduction faite des économies réalisées sur les engrais. Pour le programme FARM+CIPAN, s'ajoutent les coûts d'implantation (et d'enfouissement) des cultures intermédiaires (semences, matériel et travail). Du fait que le travail supplémentaire doit être mobilisé en période de pointe, on ne peut admettre que le coût marginal du travail familial soit nul. Le travail nécessaire a donc été valorisé au coût de l'heure salariée d'une entreprise de travaux agricoles. Ce poste est un élément

2 FARM et FARM+CIPAN. Le scénario DISC n'a pas été pris en compte dans la simulation, car il n'a pas encore réuni suffisamment de références expérimentales, tandis que les programmes FARM et FARM + CIPAN ont bénéficié de mesures expérimentales pendant deux campagnes agricoles sur 4 exploitations.
Tableau 1. - Analyse coût-efficacité comparée de deux programmes préventifs.

\begin{tabular}{|l|c|c}
\hline & FARM & $\begin{array}{c}\text { FARM+ } \\
\text { CIPAN }\end{array}$ \\
\hline Niveaux d'efficacité obtenus & & \\
Reliquat de N sous racines (kg N/ha) & 34,2 & 17,3 \\
Réduction émissions agric. (Tonnes N) & -495 & -920 \\
soit, en \% & $-37 \%$ & $-68 \%$ \\
Concentration attendue (mg/l) & $\approx 44$ & $\approx 27$ \\
Coût total des programmes & & \\
Par exploit. concernée (francs) & 4300 & 10290 \\
Par hectare concerné (francs) & 146 & 347 \\
Total plaine Bièvre (Kilo-francs) & 3490 & 8304 \\
Par habitant (francs) & 87 & 208 \\
Coût unitaire d'abattement & & $\mathbf{9 , 0 3}$ \\
(en francs par kg d'azote abattu) & $\mathbf{7 , 0 4}$ & \multicolumn{2}{|c|}{ Source : base de données 95 INRA/Rd } \\
\hline \multicolumn{2}{|c}{}
\end{tabular}

explicatif majeur de la différence de coûts constatée entre les deux programmes considérés.

Les résultats du tableau 1 suscitent les commentaires suivants :

i) L'analyse coût-efficacité montre que le programme FARM est le plus efficace et doit être adopté. En effet, il permet a priori de garantir, en moyenne, le respect de la norme de potabilité de $50 \mathrm{mg} / \mathrm{l}$ pour un coût nettement plus faible que le programme FARM+CIPAN. Cependant deux nuances doivent être prises en compte par l'agent régulateur :

- le programme FARM n'atteint la norme qu'en moyenne et ne garantit pas que la totalité des captages respectent la norme de potabilité. En effet, la variabilité constatée actuellement selon les captages (de 15 à $48 \mathrm{mg} / \mathrm{l}$ ) ou selon les communes est telle que la concentration moyenne de l'ensemble du bassin d'alimentation devrait plutôt avoisiner $35 \mathrm{mg} / \mathrm{l}$ pour que soit éliminé tout risque de dépassement de la norme dans les captages les plus pollués.

- mais en outre, l'application de ce programme serait paradoxale puisque, tout en réduisant les émissions polluantes actuelles de $37 \%$, il aboutirait néanmoins à une dégradation importante de la qualité de l'eau potable (44 mg/l) par rapport à celle qui est constatée dans les captages aujourd'hui $(32,5 \mathrm{mg} / \mathrm{l})$. Autrement dit, une dépense annuelle de $3490 \mathrm{kF}$ sur la plaine de Bièvre-Liers se solderait à terme par une dégradation de la qualité de l'eau de près de $40 \%$...

ii) A l'inverse, le programme FARM+CIPAN paraît $a$ priori trop ambitieux car il se traduirait par un coût global plus de deux fois plus élevé. Certes, ce peut être le prix de l'aversion pour le risque, car une concentration moyenne de $27 \mathrm{mg} / \mathrm{l}$ garantit avec certitude d'atteindre la norme de potabilité dans tous les captages et permet même de préserver, voire d'améliorer, la qualité actuelle de l'eau potable. Mais, en passant de FARM à FARM+CIPAN, l'accroissement des coûts de dépollution est plus que proportionnel aux gains de qualité prévisibles, ce que traduit bien l'augmentation du coût unitaire d'abattement de $7 \mathrm{~F}$ à $9 \mathrm{~F}$.

Il existe cependant une solution intermédiaire : n'appliquer ce programme qu'à une fraction des agriculteurs, ce qui permet de viser un objectif de préservation de la qualité actuelle de l'eau et de réduire les coûts de régulation [13]. 


\subsection{Evolution à long terme de la ressource et demande en eau de qualité}

En définitive, le choix à réaliser entre les programmes préventifs pose la question des critères de décision en matière de politique publique de l'eau : est-ce qu'à moyen et long terme la ressource en eau sera disponible pour les utilisateurs avec le niveau de qualité requis ? Dans le cas où elle ne le serait pas, ces utilisateurs sont-ils disposés à supporter le coût des programmes à mettre en œuvre pour préserver voire améliorer la qualité de l'eau.

\subsubsection{Simulation de la qualité de la ressource en eau à l'horizon 2015}

On a utilisé pour cette étape de la recherche un modèle de simulation du bilan ressources - besoins à I'horizon 2015, avec des pas de temps intermédiaires de 5 ans et pour 30 zones géographiques. Ce modèle, de type hiérarchique, permet une projection de la pollution future des captages si rien n'était entrepris pour préserver la qualité de l'eau potable et confronte le résultat aux besoins de trois groupes d'utilisateurs, la population, l'industrie et l'agriculture.

Les résultats montrent une évolution divergente selon les captages : plus de $35 \%$ d'entre eux continueraient à distribuer une eau de qualité avec une teneur en nitrates inférieure à $20 \mathrm{mg} /$ litre. Une proportion semblable se situerait à $20 \mathrm{ou}$ $30 \mathrm{mg} / \mathrm{l}$, ce qui est proche du niveau de qualité actuel $(32,5 \mathrm{mg} / \mathrm{l})$. Par contre, pour 11700 habitants de 6 communes (dont 2 de plus de 2000 habitants) la concentration serait proche du seuil de potabilité $(31-50 \mathrm{mg} / \mathrm{l})$ et pour 3900 habitants de 8 autres (moins de 1000 habitants) on dépasserait la norme de $50 \mathrm{mg} / 1$. Cependant, cette simulation est pessimiste puisqu'elle ne prend pas en compte la possibilité de repousser le seuil de non potabilité par des solutions palliatives (dilution, multi-alimentation) auxquelles il est largement fait recours.

\subsubsection{Capacité des consommateurs à supporter le coût des programmes préventifs}

Les consommateurs sont-ils prêts à supporter le coût des programmes préventifs pour préserver, voire améliorer, la qualité de l'eau ? Pour répondre à cette question, il faut évaluer leur " consentement à payer " via un ajustement du prix de l'eau (méthode d'évaluation contingente, MEC). Le choix méthodologique réalisé consiste à utiliser l'économie expérimentale pour limiter ou contrôler les principaux biais inhérents à une enquête classique d'évaluation monétaire. Une première enquête a été réalisée par interview en octobre 1997 auprès de 108 ménages représentatifs des consommateurs actuels et potentiels de l'eau potable dans la plaine de BièvreLiers. Les résultats font apparaitre un consentement à payer moyen de $217 \mathrm{~F}$ par ménage pour l'ensemble de l'échantillon. Une deuxième enquête, postale cette fois, a été réalisée de juin à septembre 1998 sur un échantillon de 172 ménages. Cette enquête, encore en cours de traitement, aboutit à des résultats comparables. Les refus de réponse sont faibles dans la première enquête $(11 \%)$ et très élevés dans la seconde (69\%), ce qui s'explique en grande partie par les modalités différentes d'interrogation. Un traitement économétrique, en cours, permettra de corriger les biais liés à ces différences. La partie expérimentale qui s'est déroulée parallèlement auprès de 4 groupes de 12 à 20 ménages, fait apparaître une nette infériorité des consentements à payer en situation de paiement réel par rapport aux situations hypothétiques (de l'ordre de $30 \%$ ). Au total, on n'atteint jamais le coût par habitant pour mettre en œuvre le programme proposé pour préserver la qua- lité de l'eau. L'apport des consommateurs ne suffira donc pas à financer un tel programme, ce qui signifie sans doute que le principe pollueur-payeur apparait plus équitable que le principe "pollué-payeur"... Cela pousse donc à envisager parallèlement un système d'éco-taxes ou de redevance-subvention sur les pollutions diffuses appliqué aux agriculteurs [18]. Cette perspective est en cours de discussion actuellement au niveau des ministères de l'environnement et de l'agriculture.

\section{Références}

[1] Mollard A., Vauclin M., Vachaud G., (ed.) 1998. - Agriculture durable, protection du sol et des ressources en eaux souterraines : une approche interdisciplinaire. Rapport final, Programme Environnement du Xème Contrat de Plan Etat-Région Rhône-Alpes, LTHE/CNRSINRA/R\&A, janvier, 102 p. + annexes.

[2] SCHOEN R., 1996. - Transferts de solutés dans un lysimètre en conditions contrôlées : expériences et simulations. Doctorat de l'Université Joseph Fourier-Grenoble I.

[3] Schoen R., Gaudet J.P., Bariac T., 1999 - Preferential flow and solute transport in a large lysimeter under controlled boundary conditions. J. of Hydrology, $215: 70-81$.

[4] Schoten R., Gaudet J.P., El.Rick D.E., 1999 - Modeling of solute transfer in a large undisturbed lysimeter during steady state water flux. J. of Hydrology, $215: 82-93$.

[5] Kengni L., Vachaud G., Thony J.L., Laty R., Garino B., Casabianca H., JAME P., VISCOGLIOSI R., 1994. - Field measurements of water and nitrogen losses under irrigated maïze. J. of Hydrol., Vol. 162:23-46.

[6] Normand B., 1996. - Etude expérimentale et modélisation du devenir de l'azote dans le système sol-plante-atmosphère. Doctorat de l'Université Joseph Fourier-Grenoble 1.

17] Normand B., Recous S., Vachaud G., Kengni L., Garino B., 1997. Nitrogen-15 tracers combined with tensioneutronic method to estimate the nitrogen balance of irrigated maize. Soil Sci. Soc. Am. J., Vol. 61 (5): 1508-1518.

[8] Angulo-Jaramilo R., Gaudet J.P., Thony J.L., Vaucun M., 1996. Measurement of hydraulic properties and mobile water content of a field soil. Soil Sci. Soc. Am. J., Vol. 60 (3):710-175.

[9] CARILLo-Avil.A E., 1995. - Modélisation des transferts hydriques dans le système sol-plante-atmosphère. Application à la plaine de la Bièvre (Isère). Doctorat de l'Université Joseph Fourier-Grenoble 1.

[10] Bouraoul F., Vachaud G., Haverkamp R., Normand B., 1998. - A distributed physical approach for surface-subsurface water transport modeling in agricultural watersheds. J. of Hydrol., Vol. 203:75-88.

[11] Bouraoul F., Vachaud G., Chen T., 1998. - Prediction of the effect of climatic changes and land use management on water ressources. Phys. Chem. Earth, Vol. 23 (4):379-384.

[12] LACROIX A., 1995. - Des solutions agronomiques à la pollution azotée Cahiers Agricultures (4), 333-342.

[13] Bei. F., Lacroix A.. Mollard A., 1998. - Les programmes de réduction des pollutions diffuses de l'eau entre équité et efficacité. Journées AFSE 1998 "Economie de l'Environnement et des Ressources Naturelles". Toulouse, 11-12 mai, 15 p. + annexes (Working paper INRA/R\&A n० 98-03).

[14] Leviel. B, Gabriflile B, Justes E, Mary B, Gosse G (1997). - Water and nitrate budgets in an oilseed rape-cropped rendzina soil with different amounts of fertilizer. European Journal of Soil Science (forthcoming).

[15] BRISSON N., MARY B., et allii (1998). - STICS : a generic model for the simulation of crops and their water and nitrogen balance. I. Theory and parameterization applied to wheat and corn. Agronomie, 18.

[16] LACroIX A., BALDUCHI F., 1995. - Le traitement des nitrates de l'eau potable. Bilan économique et perspectives. Techniques Sciences Méthodes, (12), Décembre, 923-929.

[17] Mol..ARD A., 1997. - Solutions curatives ou préventives à la pollution diffuse de l'eau ? une approche de la soutenabilité de la ressource en eau, Natures-Sciences-Sociétés, (5), $3: 5-21$.

[18] Le Roch C., Mollard A., 1996. - Les instruments économiques de réduction de la pollution diffuse en agriculture. Cahiers d'économie et sociologie rurales, (39-40), $2^{\circ}$ et $3^{\circ}$ trimestres, pp. 63-92.3 